\title{
HÖLDER TYPE INEQUALITIES FOR MATRICES
}

\author{
TSUYOSHI ANDO AND FUMIO HIAI
}

Abstract. We discuss Hölder type inequalites involving $\left(A^{p}+B^{p}\right)^{1 / p}$ for positive semi-definite matrices $A, B$. Matrix or trace inequalities of Hölder type as well as weak majorizations of similar type are obtained. Also we give counter-examples for expected Hölder type inequalities.

Mathematics subject classification (1991): Primary 15A45, 15A42; Secondary 15A60, 47A63.

Key words and phrases: Positive semi-definite matrices, Hölder inequality, trace inequalities, weak majorization, Oppenheim inequality.

\section{REFERENCES}

[1] T. ANDO, Concavity of certain maps on positive matrices and applications to Hadamard products, Linear Algebra Appl. 26 (1979), 203-241.

[2] T. ANDO, Majorizations, doubly stochastic matrices, and comparison of eigenvalues, Linear Algebra Appl. 118 (1989), 163-248.

[3] T. ANDO, Majorizations and inequalities in matrix theory, Linear Algebra Appl. 199 (1994), $17-67$.

[4] T. ANDO AND F. HIAI, Log majorization and complementary Golden-Thompson type inequalities, Linear Algebra Appl. 197/198 (1994), 113-131.

[5] R. BHATIA, Matrix Analysis, Springer-Verlag, New-York, 1997.

[6] K. FAN, On a theorem of Weyl concerning eigenvalues of linear transoformations. II, Proc. Nat. Acad. Sci. U.S.A. 36 (1950), 31-35.

[7] F. HANSEN AND G.K. PEDERSEN, Jensen's inequality for operators and Löwner's theorem, Math. Ann. 258 (1982), 229-241.

[8] F. HiAi AND D. PeTZ, The Golden-Thompson trace inequality is complemented, Linear Algebra Appl. 181 (1993), 153-185.

[9] T. KATO, Spectral order and a matrix limit theorem, Linear and Multilinear Algebra 8 (1979), $15-19$.

[10] H. KoSAKI, Some remarks on the positive operator $\left(a^{2}+b^{2}\right)^{1 / 2}$, unpublished notes.

[11] F. KuBo And T. Ando, Means of positive linear operators, Math. Ann. 246 (1980), $205-224$.

[12] E.H. LIEB, Convex trace functions and the Wigner-Yanase-Dyson conjecture, Adv. Math. 11 (1973), 267-288.

[13] A.W. Marshall AND I. OlkIn, Inequalities: Theory of Majorization and Its Applications, Academic Press, New York, 1979.

[14] A. OpPENHEIM, Inequalities connected with definite Hermitian forms, II, Amer. Math. Monthly 61 (1954), 463-466.

[15] M. TAKESAKI, Theory of Operator Algebras I, Springer-Verlag, New York-Heidelberg-Berlin, 1979.

[16] G. VISICK, Majorizations of Hadamard products of matrix powers, Linear Algebra Appl., to appear. 